\title{
Automatic Reduction of Vehicular Speed in Speed Restricted Areas using Wireless Technology
}

\author{
Shakeel Ahmed ${ }^{1}$ and Humaira Nishat ${ }^{2}$ \\ ${ }^{1,2}$ Department of ECE, CVR College of Engineering, Hyderabad, India \\ Email: shakeel_be@yahoo.com,huma_nisha@yahoo.com
}

\begin{abstract}
With the advanced technology being incorporated in automobiles, the acceleration in all the vehicles has been improved significantly. People using such vehicles have very little control on the upper speed limit of the vehicle while passing through small lanes, residential colonies as well as in speed restricted areas like school zones, banks, hospitals under construction areas etc. As a result the residents in such lanes had to resort to putting a number of speed breakers to avoid accidents. Erecting such speed breakers has many short term and long term effects both for the driver as well as the vehicle. Also, on main roads and highways, generally sign boards are kept which alerts the driver regarding speed limits at school zone, narrow road ahead, under construction area and so on so that the speed of the vehicle has to be reduced. But the driver of the vehicle either ignores the sign boards or the sign boards will not be visible due to the trees grown beside the road thereby covering the sign boards. Thus, there is a need to reduce the upper speed limit of vehicles. Also if this speed reduction is made possible without the intervention of the driver, the robustness of the action will be improved. Thus, this paper proposes the design of a wireless communication and control system that would control the upper speed limit of vehicles traveling on a certain road. The proposed system is simulated using Proteus 7 software and implemented using PIC Microcontroller (PIC16F877A), RF Module and other related components.
\end{abstract}

Index Terms-alarm, driver, pic micro controller, roads, signboards, wireless communication system

\section{INTRODUCTION}

Recent studies show that most of the road accidents are associated with excessive speed, carelessness of the drivers as well as the changes in the roadway i.e., frequent road construction work. Road accidents have become a big problem and a major cause of concern for the road traffic authorities, automobile industries and transport research groups. Thus, advanced driver assistance systems are incorporated in most of the vehicles today which allow the vehicle to communicate to the driver about the possibility of a collision through acoustic or visual signals produced by the vehicle. Future trends indicate that higher safety can be achieved by incorporating a large number of sensors both on the road as well as with the vehicle and through automatic driving controls. Cruise Control (CC) [3] is one of the driver assistance systems which can maintain a constant user preset speed. Adaptive Cruise Control (ACC) [1], [2], [4] which is addition to maintaining a constant user preset speed has the capability to maintain a safe distance from the preceding vehicles. But the major drawbacks of these systems are that the system is incapable of distinguishing between straight and curved parts of the road, the various speed limited restricted areas where speed has to be lowered to avoid accidents. A recently developed system known as Curve Warning Systems(CWS) uses a combination of Global Positioning System (GPS) and digital maps obtained from a Geographical Information System (GIS) which assess the threat levels of approaching a curve and alerts the driver quickly. Similarly Intelligent Speed Assistance (ISA) systems warns the driver about the inappropriate speed of vehicle using GPS and a digital road map containing information about the speed limits.

Despite all these systems, unexpected road circumstances like speed breakers, road work, road diversions, traffic congestion, etc., requires the attention of the driver while driving and have a control over the upper speed of the vehicle. Thus, some system has to be designed which would have a control or reduce the upper speed of the vehicle without the intervention of the driver in such speed restricted areas. This paper proposes the design of such a wireless communication system [9] which incorporates an RF transmitter at such speed limit zones/restricted areas and an RF receiver in the vehicles which receives the signals and in turn trigger a controller to reduce the upper speed of vehicles without the intervention of the driver.

The rest of the paper is organized as follows. Section II discusses the related work. Section III describes the proposed system with its working methodology and flowchart. Section IV discusses about the software used followed with the simulation results and section $\mathrm{V}$ gives the hardware results of the proposed system. The last section concludes the paper.

\section{RELATED WORK}

Different types of sign boards displaying the upper speed limit such as no over taking, humps, sharp curves, school zones, etc., are placed at the speed restricted areas to alert the driver by the road transport authority department as shown in fig. 2.1. It is up to the driver to follow them and take necessary action. 


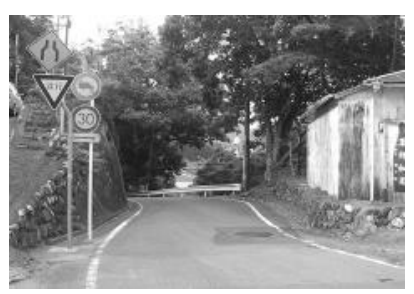

Fig. 2.1. Picture Showing Speed Limit Sign Board at Sharp Curves

In certain cases it also happens that the boards will be covered by trees that are growing beside the roads and will not be visible by the drivers as shown in fig. 2.2.

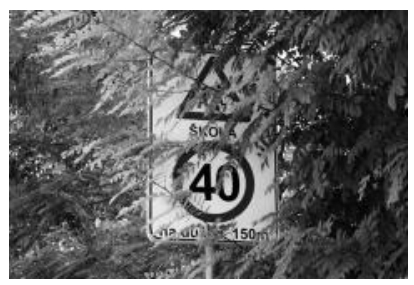

Fig. 2.2. Sign Board Covered With Trees

In order to avoid such situations one alternative is to incorporate CCS systems [3] also known as Speed Control or Autocruise or Tempomat in the vehicles. CCS is a system designed to automatically control the speed of the vehicle. It is a servomechanism system that takes over the throttle of the vehicle to maintain a steady speed asset by the driver. CCS is usually used for long drives across highways and sparsely populated roads which results in improved fuel efficiency. But when used on wet or snow and/or ice-covered roads, the vehicle may skid and results in driver losing control over the vehicle.

An alternative to CCS is Adaptive Cruise Control System (ACCS) [1], [2], [4] which is an improved cruise control. It has an automatic braking or dynamic set speed type controls. The automatic braking allows the vehicle to keep pace with the other vehicle and uses either a radar or laser setup. Some of such systems are also equipped with a feature called forward collision warning systems which warns the driver of the vehicle about the speed of the vehicles in front of it when it gets too close. Dynamic set speed type uses GPS positioning system for speed limit signs due to which the driver is relieved from the task of being careful while accelerating, deceleration and breaking in congested traffics. As the acceleration and breaking are done in a systematic way, the fuel efficiency of the vehicle is improved. But such systems enable the driver to become careless which may lead to severe accidents if the system fails.

In paper [5], authors have proposed a system to alert the driver about the speed limit at zones and to detect crashes automatically using GPS and GSM technology. The proposed work does not contribute for controlling the speed of vehicle without the intervention of the driver. Instead of controlling the vehicle speed automatically, the proposed system only alerts the driver about the speed limits and detects accidents.

In paper [6], authors have proposed a model to design an RF based speed control system for vehicles. But the system only alerts the driver by means of a buzzer and an LCD display regarding the zone in which the vehicle has entered. The system actually does not control the speed of the vehicle. By listening to the alarm, the driver himself should reduce the speed of the vehicle.

\section{Proposed SySTEM}

The proposed system is divided into two sections-the transmitter section at the poles in each zone and the receiver section at the vehicle as shown in fig. 3.1. \& fig. 3.2 respectively,

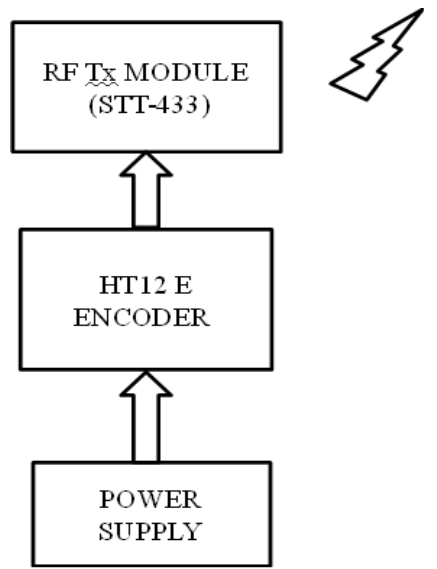

Fig. 3.1 Block diagram of the Proposed System- Transmitter Section

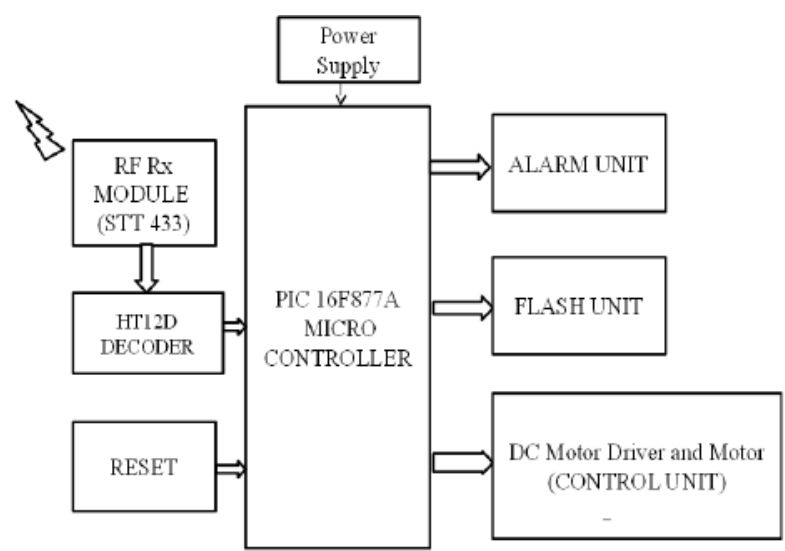

Fig. 3.2. Block Diagram of the Proposed System-Receiver Section

The transmitter section consists of a 433MHz ST-TX01ASK Hybrid RF Transmitter Module (STT-433), GT12E Encoder and a Power Supply.

ST_TX01-ASK is an effective low cost, small size transmitter module with a frequency range of $315 / 433.92 \mathrm{MHz}$, uses a supply voltage of $12 \mathrm{~V}$ and generates an output power of 4 to $16 \mathrm{dBm}$. Fig. 3.3 shows the pin diagram of $433 \mathrm{MHz}$ ASK RF Transmitter.

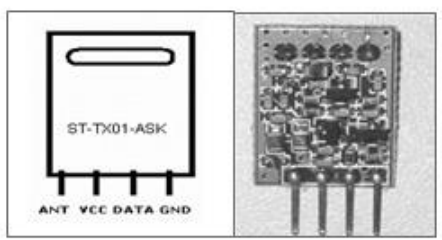

Fig. 3.3. Pin Diagram of 433 MHz RF Transmitter STT-433 
HT12E $2^{12}$ series of encoders are a series of CMOS LSIs for remote control system applications. These encoders operate on a supply voltage of $0.3 \mathrm{~V}$ to $13 \mathrm{~V}$ and are capable of encoding information which consists of $\mathrm{N}$ address bits and 12_N data bits. Each address/data input can be set to one of the two logic states. The programmed address/data are transmitted together with the header bits via an RF transmission medium. The $2^{12}$ series of encoders begin a 4word transmission cycle upon receipt of a transmission enable (TE). This cycle repeats itself as long as the TE is held low. Once TE returns to high the encoder output completes its first cycle and stops. TE is enabled by applying a low signal to the TE pin as shown in fig. 3.4.

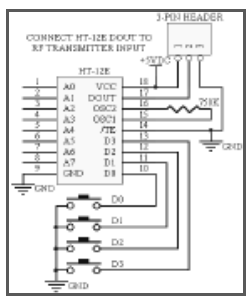

Fig. 3.4. Connections for HT12E Encoder

The receiver section comprises of a $433 \mathrm{MHz}$ ST-RX02ASK Hybrid RF Receiver Module (STR-433), HT12D Decoder, PIC Microcontroller, Power Supply, DC Motor, Motor Driver (L293D), a buzzer and an LED.

ST-RX02-ASK is an effective low cost module available at frequencies $315 / 433.92 \mathrm{MHz}$. STR-433 is ideal for short range remote control applications and requires no external RF components except for the antenna. It uses a super regenerative AM detector to demodulate the incoming AM carrier. Fig. 3.5 below shows the pin diagram of 433MHz RF Receiver STT 433.

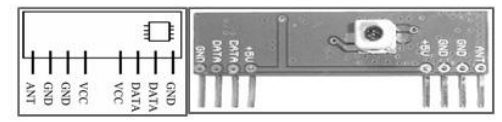

Fig. 3.5. Pin Diagram of 433 MHz RF Receiver STT-433

HT12D decoder belongs to $2^{12}$ series of decoder whose operating voltage is $2.4 \mathrm{~V}$ to $12 \mathrm{~V}$. Is is a low power, high noise immunity CMOS technology capable of decoding 12 bits of information. These are paired with Holtek's $2^{12}$ series of encoders. For proper operation, a pair of encoder/decoder with the same number of addresses and data format should be chosen. The decoder receives serial addresses and data from a programmed $2^{12}$ series of encoders that are transmitted by a carrier using an RF transmission medium. Fig. 3.6 below shows the connections for HT12D Decoder.

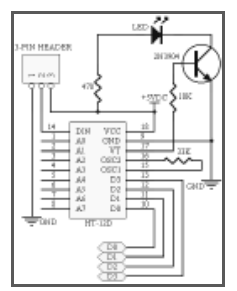

Fig. 3.6. Connections for HT12D Decoder
PIC Microcontroller (PIC16F877A) is an 8-bit microcontroller with 368 bytes of RAM, 256 bytes of EEPROM with a crystal oscillator operating at $20 \mathrm{MHz}$ and consists of 5 input/output ports viz. Port A, Port B, Port C, Port D and Port E. Each of the port can be used for a different function. Most of the ports can be used as either an input or an output port. Figure 3.7 below shows the architecture of PIC Microcontroller (PIC16F877A) and fig. 3.8 shows its pin diagram.

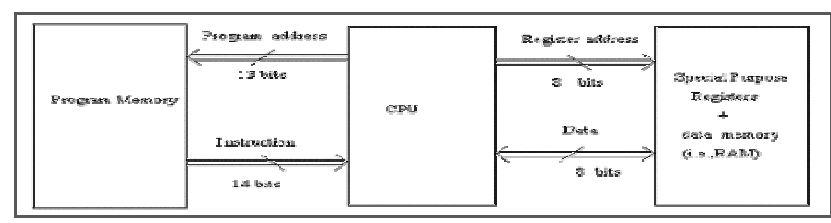

Fig. 3.7. Architecture of PIC Microcontroller (PIC16F877A)

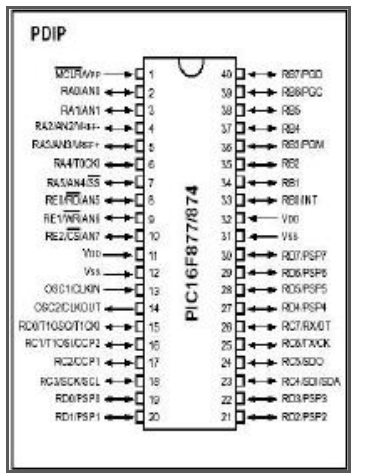

Fig. 3.8. Pin Diagram of PIC16F877A

Motor Driver (L293D): It is a dual H-bridge motor driver integrated circuit. Motor driver acts as a current amplifier by taking a low current control signal and providing a high current signal which is used to drive the motors. With L293D, two DC motors can be driven simultaneously both in forward and reverse direction. Logic 01 and 10 will rotate the motor in clockwise and anticlockwise directions respectively whereas logic 00 and 11 will stop it. Fig. 3.9a \& $3.9 \mathrm{~b}$ below shows the pin diagram and internal circuit diagram of L293D.

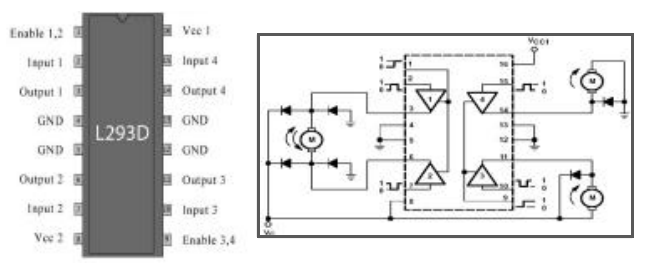

Fig. 3.9a. Pin Diagram of L293D; Fig. 3.9b. Internal Diagram

Buzzer: It is a sound source made up of piezoelectric sound component. By applying dc voltage between the electrodes of a piezoelectric diaphragm, mechanical distortions are caused due to the piezoelectric effect. To interface a buzzer the standard transistor interfacing circuit is used. Buzzer have a single tone and generates sound by using a battery as the power supply. Fig. 3.10 a \& b below shows the picture of a buzzer and its transistor interfacing respectively. 


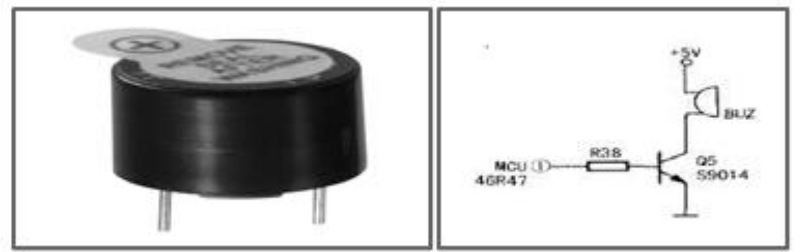

Fig. 3.10a. Picture of buzzer; Fig. 3.10b. Buzzer with transistor interfacing Circuit

LED: It is a semiconductor light source which is used as an indicator lamp in many devices.

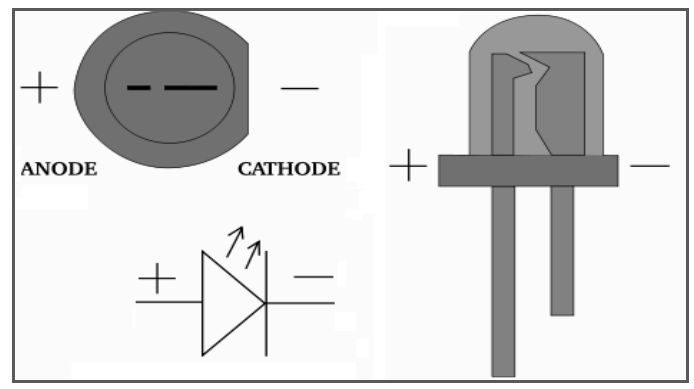

Fig. 3.11. Electrical Symbol \& Polarities of LED

\section{Working Methodology}

The proposed system works in a simplex mode i.e., one way communication. When a vehicle enters a speed restricted lane such as a school zone, under construction area and so on the signal which is continuously radiated by the ASK RF transmitter section is received and decoded by the HT12D decoder and is applied as a triggering signal to the microcontroller. The flash unit or an LED connected to the circuit glows when valid data transmission occurs from transmitter to receiver. This indicator alerts the driver about the speed limit zone. Automatically without the intervention of the driver the upper speed limit of the vehicle is reduced. This actually happens when the microcontroller unit in turn triggers the control unit. In practice the control unit has to be the Electronic Control Unit (ECU) of the vehicle which controls the flow of fuel to reduce the speed of vehicle. But for the demonstration purpose, a vehicle is fabricated whose wheels are controlled by a dc motor driver and a dc motor. Thus, the control unit is replaced by a dc motor driver and a dc motor whose rotations are controlled and reduced on receiving a signal from the RF transmitter.

Thus, without the intervention of the driver the speed can be reduced. In case the speed control unit i.e., the control unit fails the driver is alerted by a hooting mechanism or the alarm unit (buzzer). The buzzer starts sounding so that the driver has to manually take the necessary action i.e., reduce the speed. The buzzer continues to sound unless the speed is reduced. Once the vehicle comes out of the speed restricted zone, due to the absence of any radiated signal the speed of the vehicle comes back to its normal value. Fig. 3.12 gives the flowchart of the proposed system.

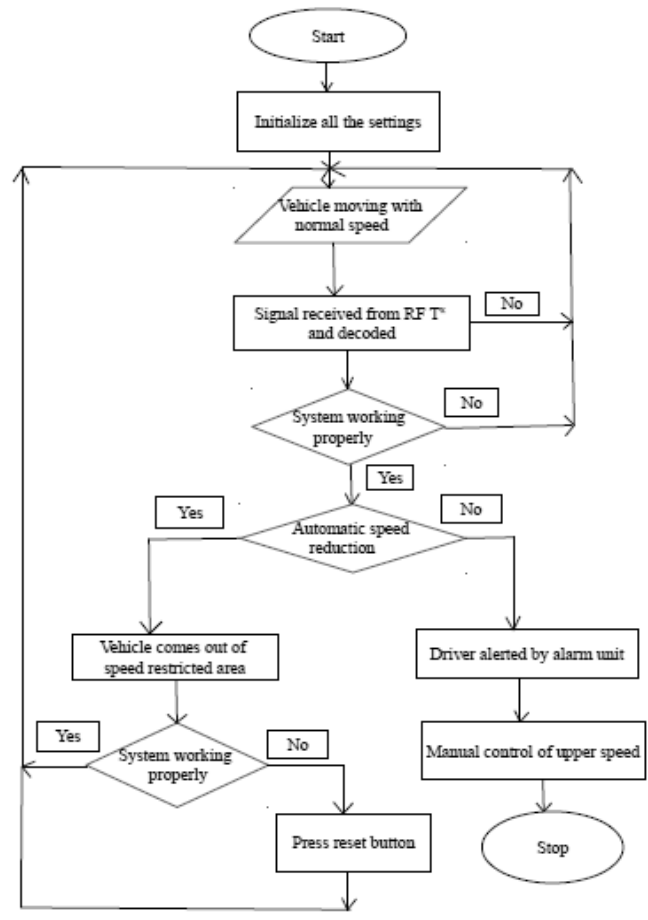

Fig. 3.12. Flowchart of the proposed system

\section{Simulation Results}

The proposed system is simulated using the Proteus 7 software.

\section{Simulation Results:}

Fig. 4.1 shows a motor moving with the normal speed in the absence of any input data i.e., no signal being radiated by the transmitter. As the vehicle enters the speed restricted area then the input data is applied i.e., when the data key switch 1 to 4 is pressed then the speed of the motor is reduced accordingly and also the LED glows. This indicates that automatically the speed of the vehicle is reduced. The flash unit i.e., the LED alerts the driver about the speed restricted area and that he has no control over the upper speed limit of the vehicle.

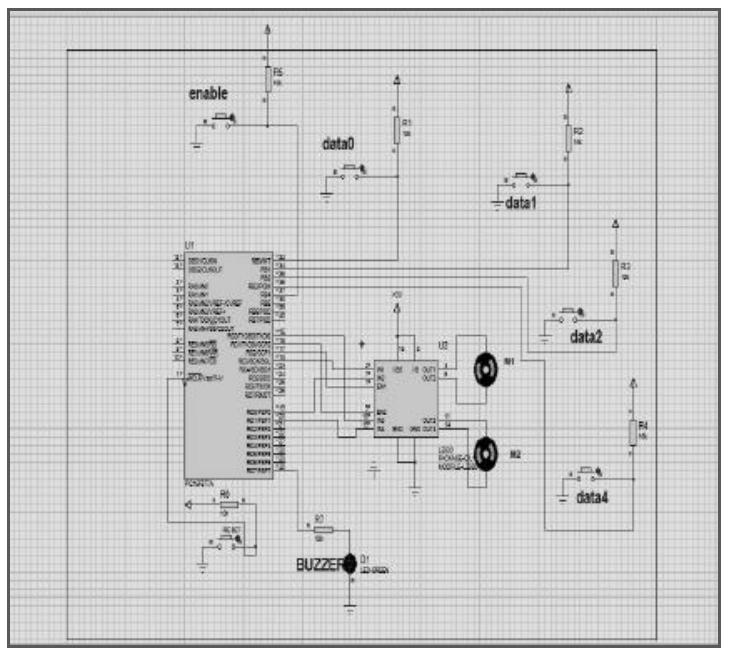

Fig. 4.1. Motor moving with normal speed when no data key is pressed 


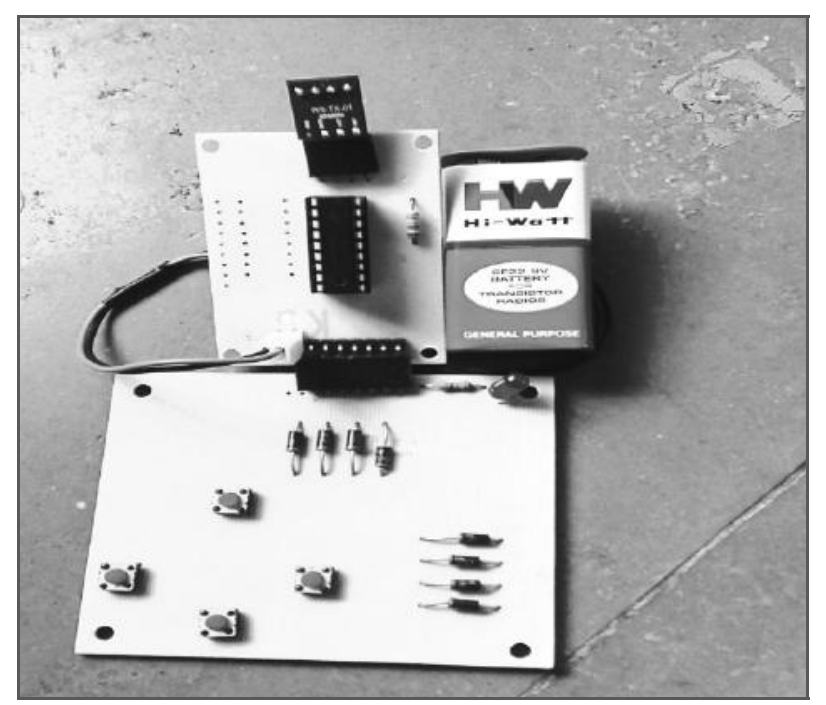

Fig. 5.1. Hardware Implemented Transmitter of the Proposed System

\section{HARDWARE RESULTS}

Fig. 5.1 shows the transmitter section of the proposed system. It comprises of a battery, four switches connected to the encoder which encodes the data and sends it through the RF transmitter.

Fig. 5.2 shows the receiver module which has to be placed and interfaced with the vehicle. The designed module reduces and limits the upper speed of the vehicle as it enters into a speed restricted area. When the switch at the transmitter side is pressed the LED at the transmitter glows and indicates that the data is being transmitted as shown in fig.5.3. Also the LED at the receiver glows indicating that the data is received and decoded by the receiver section and automatically the speed of the vehicle (here motor) is reduced.

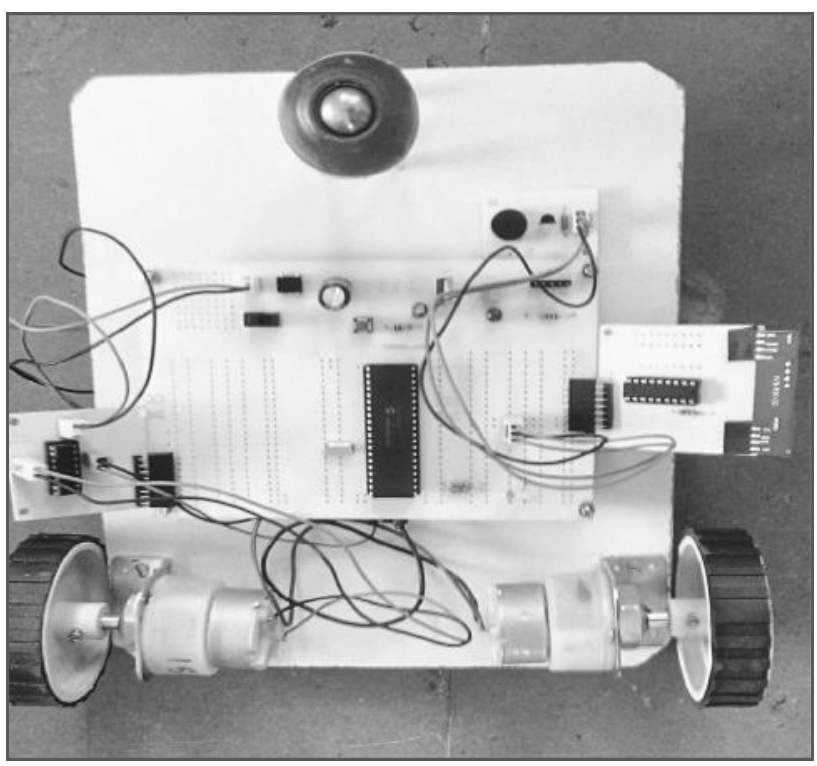

Fig.5.2. Receiver part placed in the vehicle

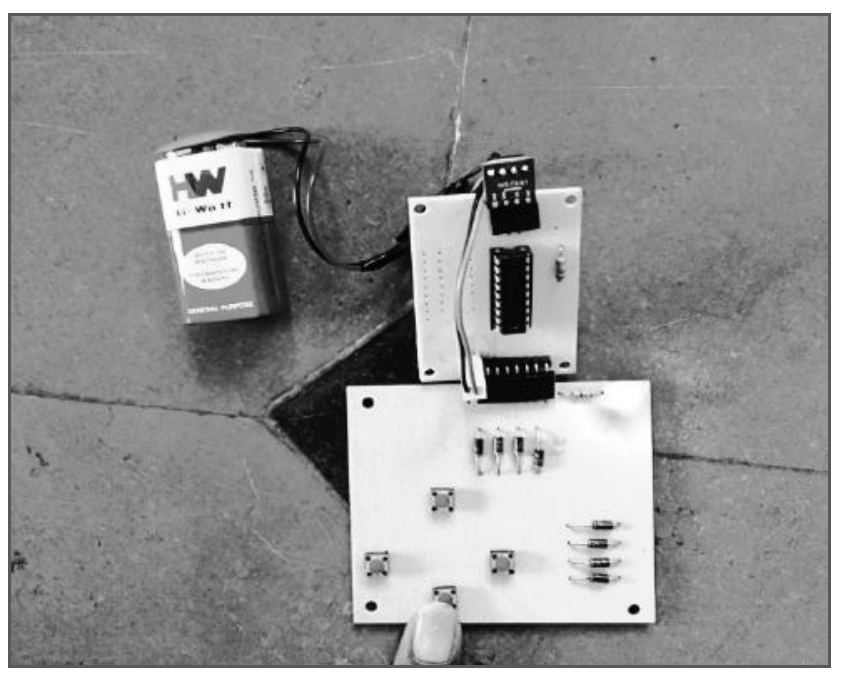

Fig. 5.3. LED Indicator Showing that One Key is Pressed

\section{ConCluSIONS}

The proposed system is realized using a dc motor and a motor driver using a PIC microcontroller instead of the ECU which controls the flow of fuel and reduces the speed of the vehicle. The proposed system can be implemented in real time and a working model can be developed which can be adapted by the automobile manufacturers and the road transport authorities. This system can prevent road accidents to a large extent as it avoids over speeding in the speed limit zones. It also takes care of wear and tear conditions of vehicles engine and driver health conditions as it is possible to avoid erecting speed breakers in lanes and speed restricted zones. Thus, the system can be easily implemented which ensures maximum safety not only to drivers but also to the passengers and pedestrians.

\section{REFERENCES}

[1] Ardalan Vahidi, Azim Eskandarian, "Research Advances in Intelligent Collision Avoidance and Adaptive Cruise Control", IEEE Transactions on Intelligent Transportation Systems, vol.4, no.3, pp. 143-153, Sept. 2003.

[2] G.N.Bifulco, F.Simonelli, R.D Pace, "Experiments Towards Human Like Adaptive Cruise Control", Proc. IEEE Intelligent Vehicles Symposium, Eindhoven, pp.919-928, 2008 .

[3] F.A.Arvind Raj R., S.B. Sandhiya Kumar and T.C. Karthik S., "Cruise Control Operation from Zero to Preset Speed Simulation and Implementation", International Journal of Information and Education Technology (IJIET), vol.1, no.1, pp. 9-14, April 2011.

[4] Shigeharu Miyata, Takashi Nakagami, Sei Kobayashi, Tomoji Izumi, Hisayoshi Naito, Akira Yanou, Hitomi Nakamura and Shin Takehara, "Improvement of Adaptive Cruise Control Performance", EURASIP Journal on Advances in Signal Processing, vol. 2010, pp. 1-8, Dec. 2010 .

[5] D.Narendra Singh, Ravi Teja ch.v, "Vehicle Speed Limit Alerting and Crash Detection System at Various Zones", 
International Journal of Latest Trends in Engineering and Technology (IJLTET), vol.2 Issue1, pp.108-113, Jan 2013.

[6] Ankita Mishra, Jyothi Solanki, Harshala Bakshi, Priyanka Saxena, Pranav Paranjpe, "Design of RF Based Speed Control System for Vehicles", International Journal of Advanced Research in Computer and Communication Engineering (IJARCCE), vol.1 Issue 8, Oct 2012.

[7] V.V.Sivaji, M.Sailaja, "Adaptive Cruise Control Systems for Vehicle Modeling Using Stop and Go Manoeuvres", International Journal of Engineering Research and Applications (IJERA), vol.3, Issue 4, pp.2453-2456, JulyAug 2013.

[8] M.Ben Swarup, M. Srinivasa Rao, "Safety Analysis of Adaptive Cruise Control System Using FMEA and FTA",
International Journal of Advanced Research in Computer Science and Software Engineering (IJARCSSE), vol.4 Issue 6, pp.330-337, June 2014.

[9] V.Abinayaa, Anagha Jayan, "Case Study on Comparison of Wireless Technologies in Industrial Applications", International Journal of Scientific and Research Publications, vol.4 Issue 2, pp.1-4, Feb 2014.

[10] The "8051 Microcontroller and Embedded Systems" by Muhammad Ali Mazidi and Janice Gillispe Mazidi, Pearson Education.

[11] PIC Microcontroller Manual - Microchip

[12] PIC16F877A Data sheet 